CrossMark \& click for updates

Cite this: Soft Matter, 2015, 11,5180

Received 14th April 2015, Accepted 5th June 2015

DOI: $10.1039 / \mathrm{c} 5 \mathrm{sm} 00877 \mathrm{~h}$

www.rsc.org/softmatter

\section{Photophysical and photochemical processes in 3D self-assembled gels as confined microenvironments}

\begin{abstract}
Raúl Pérez-Ruiz $\dagger^{\star^{a}}$ and David Díaz Díaz $\dagger^{\star a b}$
Numerous challenging transformations take place in nature with high efficiency within confined and compartmented environments. This has inspired scientists to develop spatially micro- and nanoreactors by 'bottom-up' approaches in order to improve different processes in comparison to solution, in terms of kinetics, selectivity or processability. In this respect, investigation of photophysical and photochemical processes in soft gel materials has recently emerged as a new and promising research field oriented towards expanding their applications in important areas such as photovoltaics, photocatalysis and phototherapy. Herein, we summarize the few examples dealing with intragel photo-induced physical and chemical processes involving embedded reactants that do not participate in the assembly of the gel network.
\end{abstract}

\section{Introduction}

In nature, a large number of challenging chemical transformations take place in confined environments such as enzymes and cells. Over the past few decades, this has inspired scientists to develop spatially confined micro- and nanoreactors by

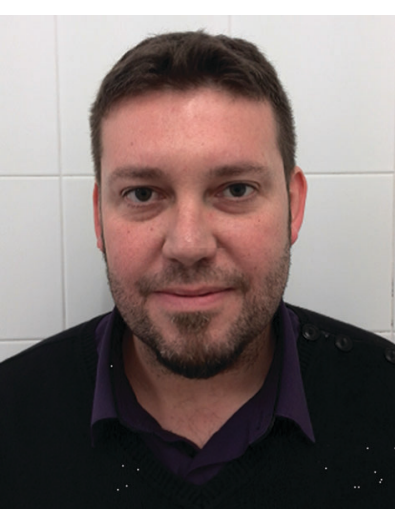

Raúl Pérez-Ruiz
Dr Raúl Pérez Ruiz (born 1976, Valencia) obtained his BSc in chemistry at the University of Valencia (2001). In 2006, he received his $P h D$ in chemistry with honors under the guidance of Prof. Miguel Angel Miranda at the Technical University of Valencia (UPV). After six months at the University of Athens (Greece) as post-doc (2006-2007), he was working as a postdoctoral scientist with Prof. Axel G. Griesbeck at the University of Cologne (2007-2008). He rejoined to the Department of Chemistry at UPV with a postdoctoral position under the supervision of Prof. Maria Consuelo Jiménez (2009-2014). Currently, he is developing his scientific career at the University of Regensburg as experience postdoctoral researcher. He is the recipient of the Alexander von Humboldt Fellowship Award, Juan de la Cierva Fellowship and IEF Marie Curie Fellowship. His actual research is focused on organic photochemical processes and molecular spectroscopy.

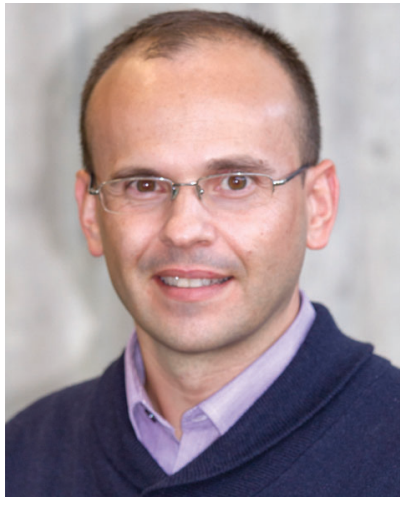

David Díaz Díaz

Prof. Dr David Díaz Díaz (born 1974, Tenerife) received his $P h D$ in chemistry with honors under the supervision of Prof. Victor S. Martin at the University of La Laguna. In 2002, he joined Prof. Finn's group as postdoc at The Scripps Research Institute (San Diego, US). Since 2006, he has held various positions in academia and industry ('Ramón $y$ Cajal' Researcher, Autonomous University of Madrid (UAM), Spain, 2006; Sr. Chemist, The Dow Chemical Company, Switzerland, 2007; Tenured Scientist, CSIC, Spain, 2009; Alexander von Humboldt Experienced Researcher, University of Regensburg, Germany, 2010). In 2013, he was named recipient of the prestigious DFG Heisenberg Professorship. Among several international awards, he has received the Young Investigator Award from the Polymer Network Group (Japan, 2014) and is Editor-in-Chief of Gels. His main research interest focuses on the development of advanced functional soft materials. 
self-assembly of low molecular weight building blocks via noncovalent interactions including hydrogen bonding, $\pi$-stacking, van der Waals, electrostatic, hydrophobic and metal-ligand interactions. ${ }^{1}$ Within this context, substantial efforts have been devoted to alter the selectivity of chemical reactions and activate new reaction pathways by arranging the reactants in a number of heterogeneous and organized media. ${ }^{2}$

In particular, the fields of photochemistry and photocatalysis have been also rich beneficiaries of this concept. ${ }^{3-6}$ In general, large active reaction areas, the possibility of tailoring additional functionalities to the microreactor environment, the reduction of overheating and overconcentration effects and reusability are some of the advantages of the spatial confinement of photochemical processes. In such kinetically controlled reactions, properties such as the absorption of light, generation of elementary redox intermediates, frontier molecular orbital energy, lifetime of excited species, rate of competitive steps and adsorption-desorption of chemical entities are largely affected by the nature of the reaction environment. ${ }^{3,7}$ The state of the art on confined photo-induced reactions include the use of mesoporous inorganic materials ${ }^{8}$ and organized molecular assemblies ${ }^{9}$ such as microemulsions stabilized with colloidal photocatalysts, ${ }^{10}$ micelles, ${ }^{11}$ vesicles modified with semiconductor nanoparticles, ${ }^{12}$ polyelectrolyte multilayered capsules ${ }^{13}$ and photocatalyst-loaded liquid foams. ${ }^{14}$

However, the use of supramolecular viscoelastic gels, ${ }^{15}$ made from low molecular weight (LMW) compounds, as reaction vessels for photophysical and photochemical processes has been only scarcely explored despite their potential as nanoreactors. ${ }^{16}$ In general, high specific surface areas, remarkable diffusion properties, reversibility, structural/functional tunability and responsiveness to multiple stimuli are some of the major features of these fascinating materials. ${ }^{15}$ Such properties offers a versatile platform to overcome some major disadvantages of other confined media including (a) high sensitivity to deactivation by irreversible adsorption or steric blockage of heavy secondary products, (b) difficulty to design the proper geometries of the components and, therefore, to exploit shape selectivity, (c) impossibility of using nonelastic microporous for the synthesis of bulky molecules and (d) competitive binding between reactive and non-reactive species, which makes difficult the recycling of the catalyst and/or the separation of the product. ${ }^{17,18}$ Moreover, tailoring the interfacial physicochemical properties of the gel network may allow a more precise control of the reactivity of embedded molecules and enhance their degrees of freedom in comparison to those in the solution state, what may at first seem counterintuitive. ${ }^{7}$

Herein, we discuss a growing research area focused on the use of fibrillar gel matrices as versatile reaction vessels to carry out photophysical and photochemical processes of physically embedded reactants, giving similar or superior results than those obtained in solution state. Well-known photopolymerization of gels containing polymerizable groups ${ }^{16}$ and the use of gelator systems with intrinsic photoactivity ${ }^{19}(e . g$., chromophoric gelators ${ }^{20}$ ) are out of the scope of this manuscript.

\section{Photophysical processes in organogels}

Biewer and co-workers ${ }^{21}$ pioneered the study of photophysical processes that can take place inside gel materials. These authors succeeded, for the first time, in measuring the rates of thermal decay of different compounds embedded in organogels. In particular, they studied a series of $1^{\prime}$-substituted 6-nitro-3', $3^{\prime}$-dimethylspiro( $2 H-1$ benzopyran-2, $2^{\prime}$-indoline) compounds with different functionalities. Interestingly, significant retardation of the decay rate, in comparison to homogeneous solution, was observed for the succinyl ester spiropyran derivative $\mathbf{1}$ physically incorporated in a supramolecular gel made from 4-tert-butyl-1-phenylcyclohexanol (3, BACO1) (Scheme 1). A unique mode of interaction accounting for the intercalation of the succinyl group of $\mathbf{1}$ in the gelling agent was proposed to explain these results. The lifetime of the colored photomerocyanine 2 was increased by over 300 -fold in the organogel in comparison to solution with a succinyl ester functionality present. Moreover, structurally similar spiropyrans without the succinyl ester functionality showed relatively little change in the lifetime of 2 .

Luis and co-workers ${ }^{22}$ studied the role of self-assembled fibrillar organogels made from LMW peptidomimetic gelator 5 (Scheme 2) on the rates of an electron transfer (ET) photoreaction. Specifically, a comparison of the anthracene (6) fluorescence quenching by amines in both, solution and organogel medium were performed. From Stern-Volmer analysis of the emission, quenching rate constants were very similar in both media, being a diffusion-controlled process in the self-organized medium using the appropriate donor. The same group investigated later ${ }^{23}$ the interaction of naproxen (4), a non-steroidal anti-inflammatory drug, with a self-assembled fibrillar gel network made from 5 by means of time-resolved fluorescence spectroscopy (Scheme 2). In this work, emission lifetime $\left(\tau_{\mathrm{F}}\right)$ of $\mathbf{4}$ in the presence of the organogel $\left(10.3 \mathrm{~g} \mathrm{~L}^{-1}\right)$ was satisfactory fitted by a biexponential decay and two $\tau_{\mathrm{F}}$ values were found to be $8.3 \mathrm{~ns}(93 \%)$ and $1.16 \mathrm{~ns}$ (7\%) that were attributed to free 4 and 4 in the vicinity of the fibers formed by the organogel, respectively. It is important to mention that this interaction was accompanied by a destabilization of the corresponding supramolecular organogel.

More recently, the same group ${ }^{24}$ used the LMW gelator 5 as a matrix to embed CdSe/ZnS quantum dots (QDs) with consequent fluorescence induction but without detriment of the thermal and optical properties of the parent gel. Very interestingly, the presence of the QDs decreased the critical gelation concentration
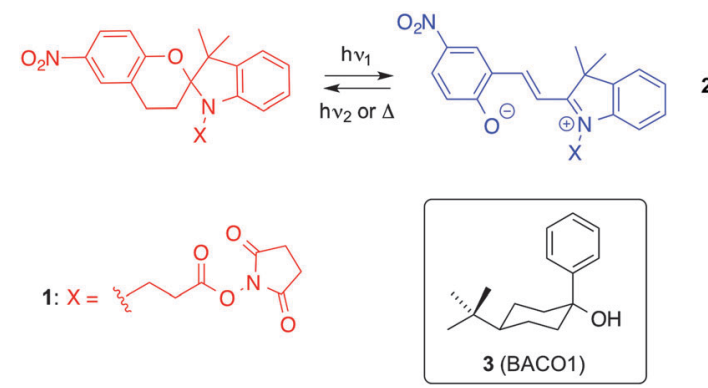

Scheme 1 Photochromism of spiropyran 1 and LMW gelator $3^{21}$ 


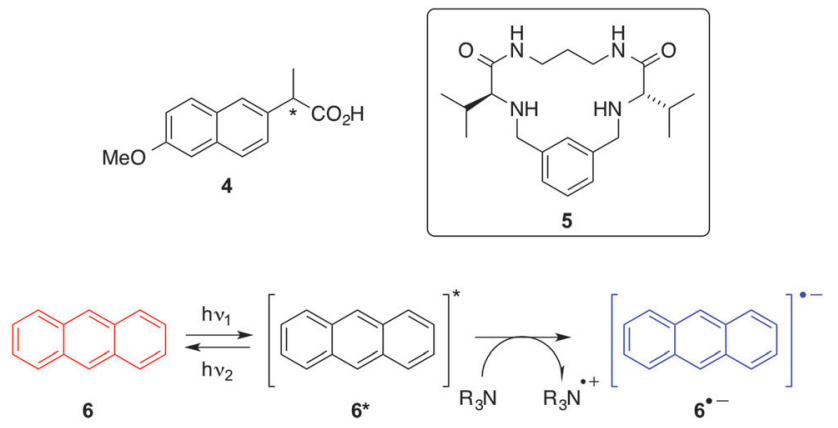

Scheme 2 Structures of naproxen (4), peptidomimetic organogelator 5 anthracene (6) and reduction of its excited state by amines. ${ }^{22}$

necessary to form stable organogels. A full photophysical study highlighted a remarkable example of organogel-nanoparticle symbiosis. Thus, core-shell QDs maintained their photophysical properties in the gel medium (regardless the gelator concentration), whereas a significant increase of the fluorescence intensity (i.e., up to $528 \%$ ) and the average lifetime (i.e., up to 1.7) was observed for the core QDs embedded in the gel (Scheme 3). Moreover, some of these composites have shown fluorescence sensitivity towards nitric oxide ranging from 0.05 to 0.5 (vol\%) without disruption of the semi-solid state of the supramolecular gel. ${ }^{25}$

On the other hand, photon upconversion (UC) based on triplet-triplet annihilation (TTA) between organic molecules is one of the most attractive wavelength conversion technologies, which can be performed with low-intensity and non-coherent light, such as ambient sunlight. ${ }^{26}$ This phenomenon, TTA-UC, implies the association of multistep photochemical events (Scheme 4). After absorption of low energy photons $\left(h \nu_{1}\right)$, triplet excited state $\left(\mathrm{T}_{1}\right)$ of the donor (sensitizer) is produced by intersystem crossing (ISC) from the singlet excited state $\left(\mathrm{S}_{1}\right)$. Subsequently, triplets of the acceptor (emitter) are populated by triplet-triplet energy transfer (TTET) from the triplets of the donor (Dexter mechanism). When two acceptor molecules in their triplet sates are capable to collide during their lifetimes, a higher singlet energy level is formed by triplet-triplet annihilation (TTA) and, consequently generates delayed upconverted fluorescence $\left(h \nu_{2}\right)$. This conversion of low power energy light into higher energy has been successfully applied in diverse

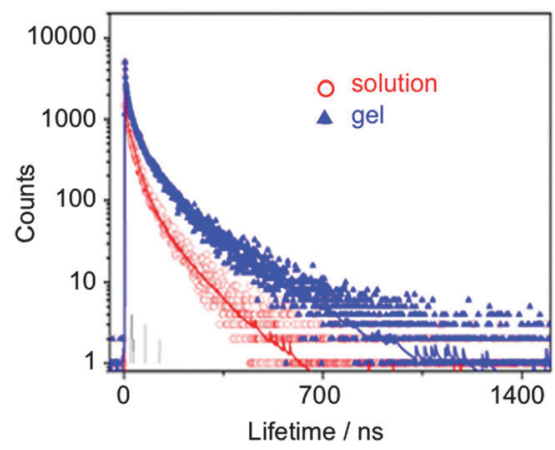

Scheme 3 Fluorescence decay curves of QDs in toluene and toluene organogel containing $5\left(3.2 \mathrm{~g} \mathrm{~L}^{-1} ; \lambda_{\mathrm{em}}=530 \mathrm{~nm}\right)$. Adapted with permission from ref. 24. Copyright (C) 2015 American Chemical Society.

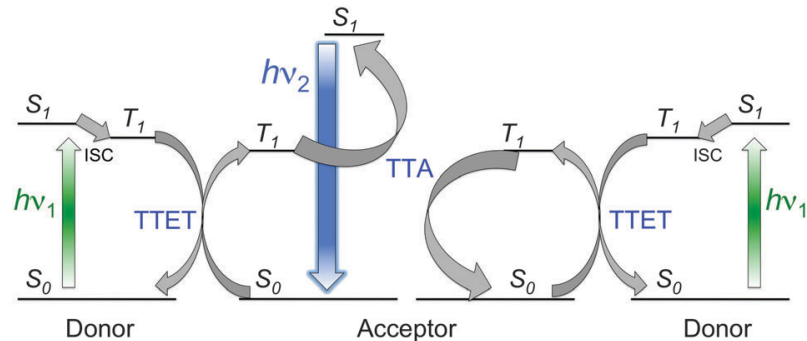

Scheme 4 Outline of the TTA-UC process showing the involved energy levels. Adapted with permission from ref. 33. Copyright (C) 2015 American Chemical Society.

scientific areas such as photovoltaics, photocatalysis, bioimaging or phototherapy. ${ }^{27-30}$

It is well assumed that the best efficient TTA-UC systems have been performed in homogenous solutions due to fast diffusion of excited molecules. Within this context, the development of easily processable materials such as viscoelastic gels (i.e., where liquid molecules are trapped in the interstices of fibrillar networks) that could be as efficient as a liquid composition remains a big challenge and it would provide new research areas in terms of applicability. In a seminal work, Simon and co-workers have recently developed new hybrid organogels containing the UC chromophore pair Pd(II) mesoporphyrin IX (PdMesoIX) and 9,10-diphenylanthracene (DPA) using DMFDMSO as solvent system. In this case, the 3D polymer gel network was formed by covalently cross-linking poly(vinyl alcohol) (PVA) with hexamethylene diisocyanate (HMDI) (Scheme 5). The results demonstrated that these gels displayed an efficient green-to-blue UC with UC quantum yields of $>0.6$ and $14 \%$ under ambient and oxygen-free conditions, respectively. ${ }^{31}$

This background could be easily extended to other materials based on other solvents, gel networks (i.e., chemical or physical gels) and/or chromophore pairs. Thus, Schmidt and co-workers have also published a highly efficient photochemical UC in a quasi-solid physical organogel based on 1,3:2,4-bis(3,4-dimethylbenzylidene) sorbitol (DMDBS) as LMW gelator, tetralin as solvent, and the pair palladium tetraphenylporfirin and DPA as photochemically active compounds. ${ }^{32}$ The experimental results

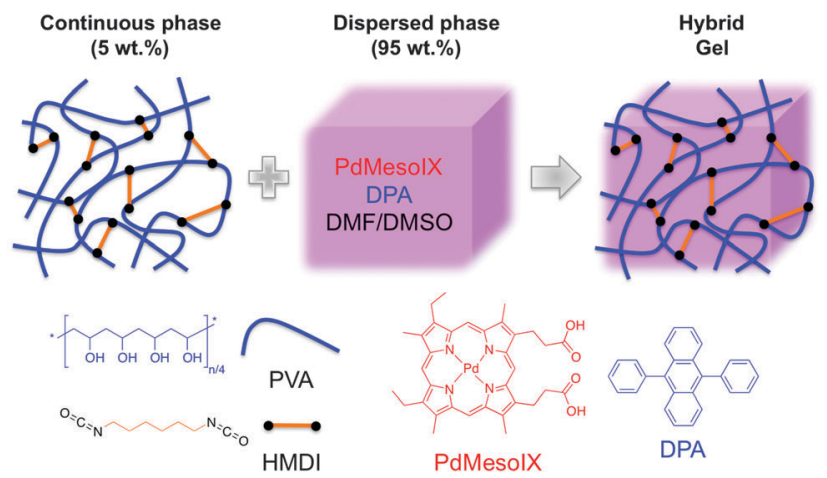

Scheme 5 Graphic representation of the composition of UC hybrid organogels used by Simon and co-workers. Adapted with permission from ref. 31 . Copyright (C) 2015 Royal Chemical Society. 


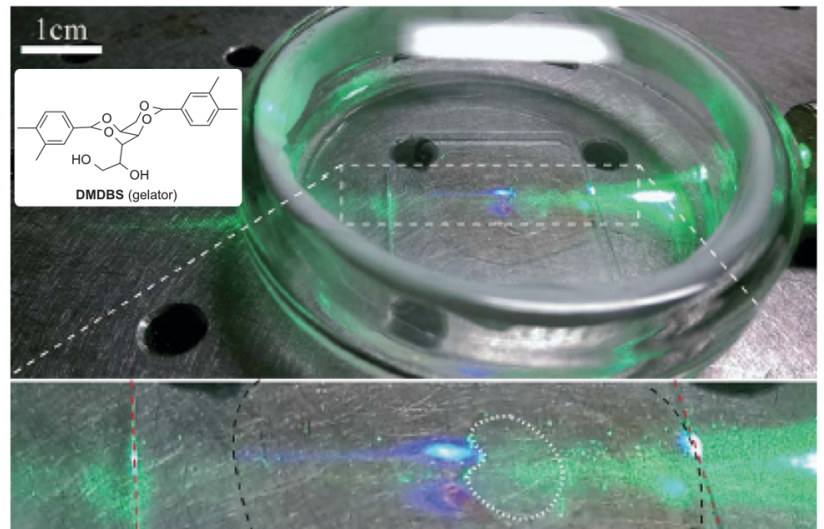

Scheme 6 Photograph of a glass slide spin-coated with PUC organogel (top) and partial zoom (bottom). The slide was submersed in an oxygenscavenging water layer. When $532 \mathrm{~nm}$ light is applied to slide, PUC is clearly visible along the path of the laser in the region covered by the film (surrounded by the black dashed line, bottom). When a patch of the gel layer was removed (dotted white outline, bottom), UC light was not visible in that region. Adapted with permission from ref. 32. Copyright (C) 2015 American Chemical Society.

have confirmed the identical TTA-UC efficiency in the quasisolid gelated sample and in a comparable liquid composition $\left(\Phi_{\text {TTA }}=0.07\right)$. As a matter of fact, this finding emerges as a suitable strategy for potential applications in devices where the gel matrix needs to be processed as a thin film (Scheme 6), photovoltaics, and photocatalytic water splitting.

Moreover, an efficient TTA-UC process was also recently developed using supramolecular organogel matrices based on $N, N^{\prime}$-bis(octadecyl)-L-boc-glutamic diamide (7) as LMW gelator (Scheme 7). ${ }^{33}$ In this case, intense UC emission was detected

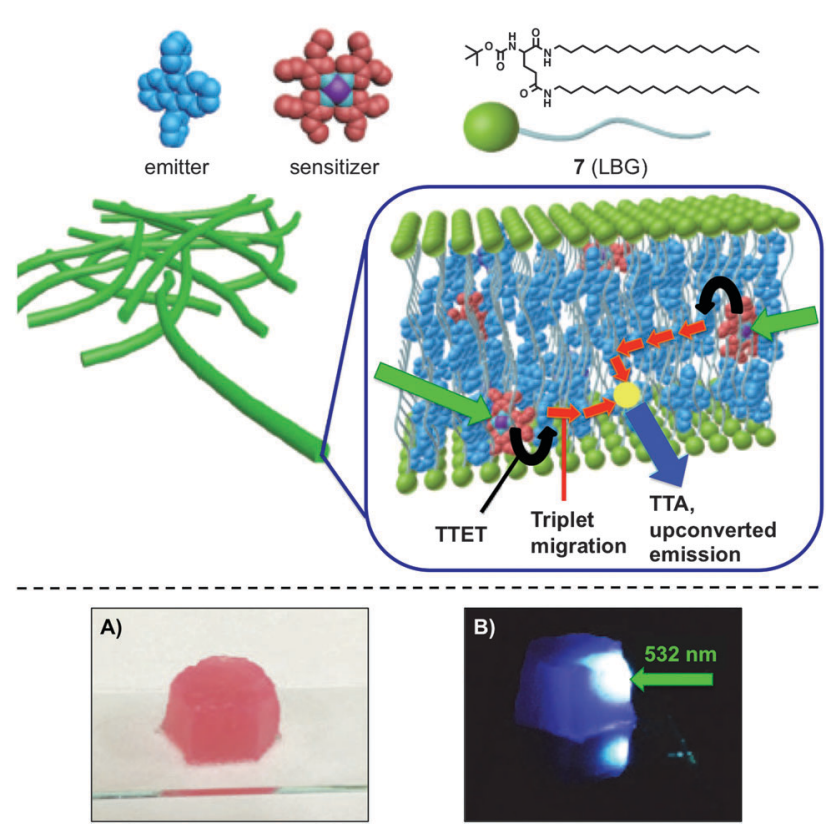

Scheme 7 Schematic representation of the structural unit of the upconversion ternary gel system (top), and pictures of the gel shaped in a mould under (A) white light and (B) $532 \mathrm{~nm}$ green laser (bottom). Adapted with permission from ref. 33. Copyright (C) 2015 American Chemical Society. from different donor (sensitizer)-acceptor (emitter) pairs in organogels made of 7 even under air-saturated condition, which undoubtedly represents a major achievement in this emerging field.

These TTA-UC molecular systems were formed by spontaneous accumulation of donor and acceptor molecules in the gel nanofibers, which were apparently stabilized by developed hydrogen bonded networks. These molecules, pre-organized in the gel network, showed efficient transfer and migration of triplet energy, as revealed by a series of spectroscopic, microscopic, and rheological characterizations. Interestingly, efficient TTA-UC was achieved even under excitation power lower than the solar irradiance. These observations reveal the adaptive feature of host fibrillar gel networks that allowed efficient and cooperative inclusion of donor-acceptor molecules while maintaining their supramolecular structural integrity. Moreover, the air-stable TTA-UC in the supramolecular gel was observed for a wide combination of donor-acceptor pairs, which enabled efficient near IR-to yellow, red-to-cyan, green-to-blue, and blueto-UV wavelength conversions (Scheme 8).

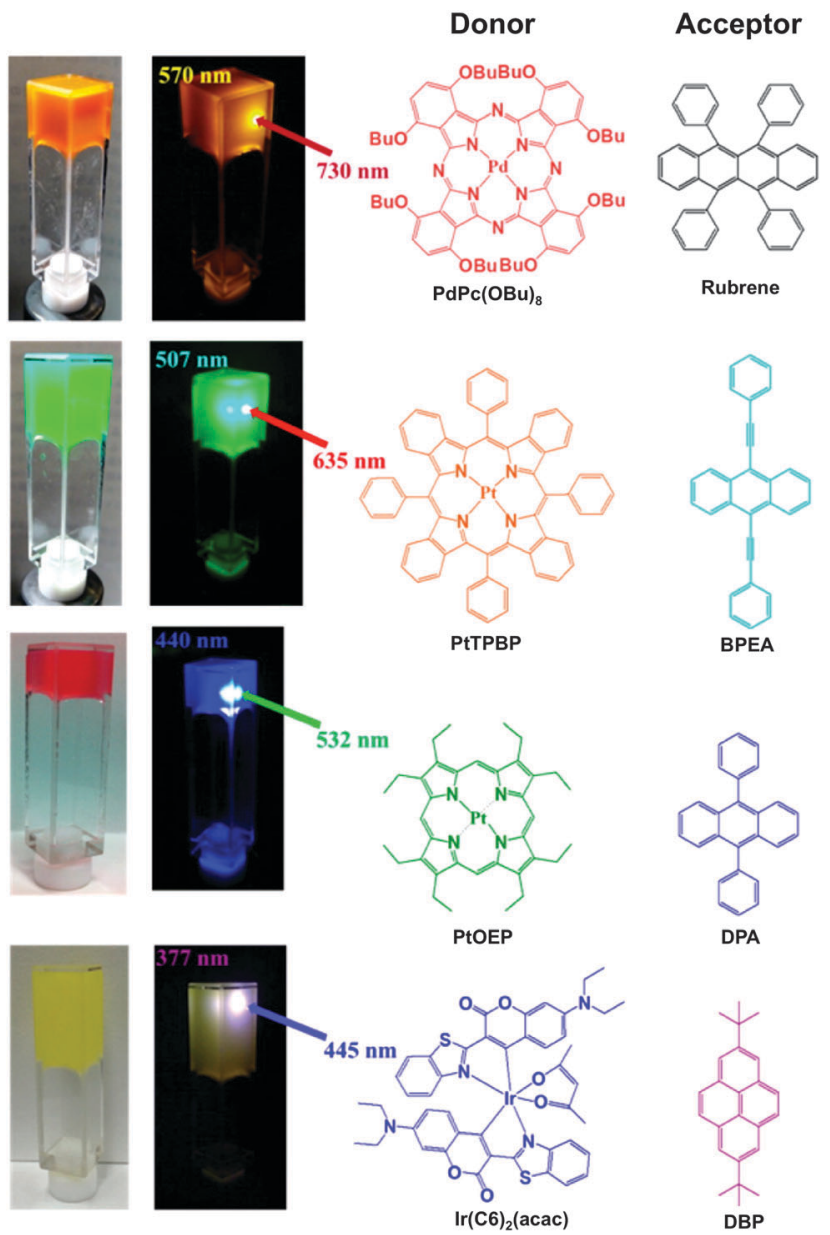

Scheme 8 Photographs of ternary UC gels in air-saturated DMF before and after irradiation. Short pass filters were used to remove the scattered excitation lights. Chemical structures of donor and acceptor pairs used in this work are also shown. Adapted with permission from ref. 33. Copyright (C) 2015 American Chemical Society. 


\section{Intragel photochemical reactivity}

The use of gel networks as confined systems to influence the course of organic photo-induced reactions has been practically unexplored and only few examples can be found in the recent literature. These reports include either photodimerization or photooxidation processes or are discussed next.

\section{Photodimerization}

Although anthracene photodimerization is one of the oldest known photochemical reactions, it still remains one of the most studied processes in different hosts or template molecules. In this respect, Shinkai and co-workers reported the first example in which a binary gelator containing a 2-anthracenecarboxylic acid (2Ac) was investigated, resulting in a very high degree of stereoselectivity under simple conditions. ${ }^{34}$ Subsequently, they developed a new class of binary organogelator based on 2Ac, attached non-covalently with the gelator counterpart containing a 3,4,5-tris(n-dodecyloxy)-benzoylamide backbone. ${ }^{35}$ Different degrees of stereoselectivity for different systems were observed when 2Ac-containing gel samples were irradiated. Furthermore, the nature of both gelator and solvent was found to play an important role in the stereochemistry of the obtained photodimers. Ongoing with this research, the same group demonstrated that a high degree of chiral induction could be also achieved and controlled precisely by the 1D supramolecular gelator assembly, which would path the way for future research in this direction. ${ }^{36}$

Although photodimerization of acenaphthylene (8) (Scheme 9, top), which usually yields a mixture of syn (9) and anti (10) dimers along with minor oxidation products, had been previously studied in confined media such as zeolites or micellar solutions. However, Maitra and co-workers investigated the process, for the first time, inside supramolecular hydrogels (i.e., based on LMW bile acids 11-14, Scheme 9, bottom) as potential photochemical nanoreactors. ${ }^{37}$ The results showed that the photodimer ratio $(\mathbf{9}: \mathbf{1 0})$ obtained for the reaction performed in the gel-bound phase

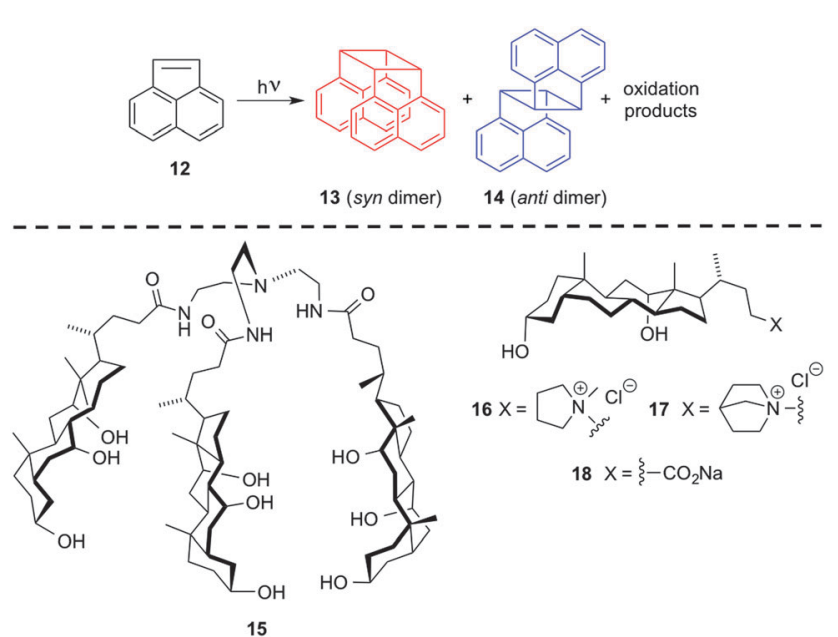

Scheme 9 Photodimerization reaction of acenaphthylene (8) (top) and structure of hydrogelators 11-14 (bottom). ${ }^{37}$ was 3-10 higher in comparison to either micellar solutions or water. Very remarkably, the product selectivity was found to be highly dependent on the rheological properties of the hydrogels, obtaining higher storage modulus $\left(G^{\prime}\right)$ in the most rigid gels (i.e., $13>12>14>11)$. Fluorescence experiments showed different intensity of the ACN-excimer band in the gel-bound state and in micellar solution, pointing out the existence and a key role of different molecular interactions patterns during the photochemical process.

More recently, Yadav and co-workers ${ }^{38}$ have also reported the use of an organogel (i.e., made from enantiomerically pure 3,4-O-isopropylidenearabinose) as a reaction vessel for the production of a bicyclo[3.2.1] octanone via photo-induced electron transfer (PET) upon irradiation at $300 \mathrm{~nm}$. Both yield and reaction time were similar to those obtained in solution state. ${ }^{39}$ Importantly from the recycling point of view, the gelator could be also recovered from the mixture after column chromatography.

\section{Photosensitized isomerization}

Yang, Trotta, Inoue and co-workers ${ }^{40}$ have recently reported the preparation of hydrogels made of polymers based on cyclic nigerosylnigerose (CNN; a saucer-shaped tetrasaccharide) that act as a "nanosponge" (NS). The CNN-NSs were prepared by crosslinking CNN with pyromellitic dianhydride (PDA) at different ratios. These polymeric materials effectively worked as chiral supramolecular sensitizers (Sens* in Scheme 10, bottom) and mediated the enantiodifferentiating photoisomerization of $(Z)$-cyclooctene to chiral (E)-isomer (Scheme 10, top). Note that CNN is a "pseudo" host lacking a confining cavity to fully accommodate the guest molecule. Very interestingly, the product's ee was dramatically controlled by the phase evolved. Indeed, the ee of the $(E)$-isomer was low in solution and suspension, but rapidly increased at the

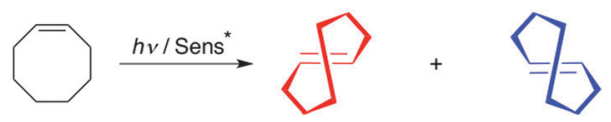
Z-cyclooctene
(R)-(-)-E-cyclooctene (S)-(+)-E-cyclooctene

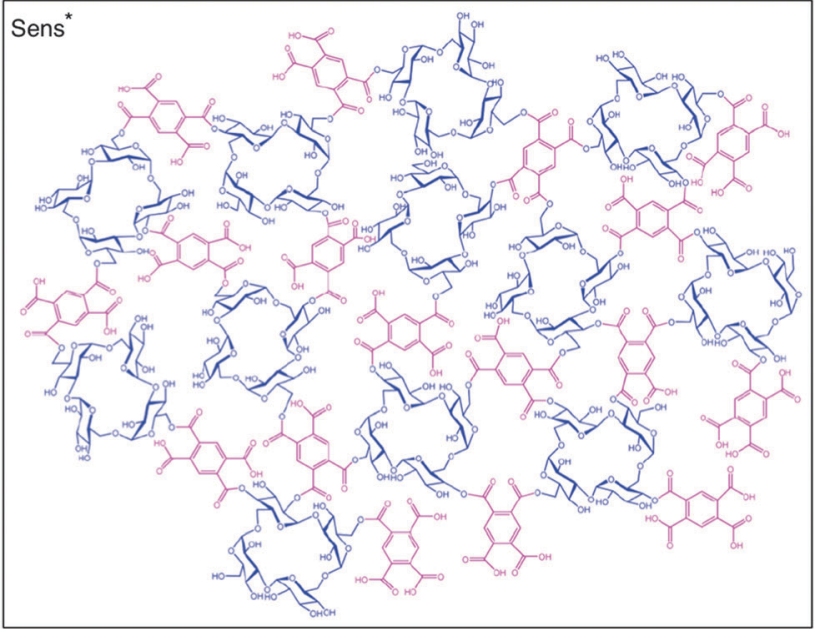

Scheme 10 Enantiodifferentiating photoisomerization of (Z)-cyclooctene to chiral (E)-cyclooctene (top) mediated by CNN-NSs (bottom). ${ }^{40}$ 
phase border between flowing gel and rigid gel. These results are very stimulating because they clearly reveal the beneficial role of the chiral void space formed upon gelation of the crosslinked polysaccharide on the outcome (i.e., chirality transfer) of supramolecular photochirogenic reactions. The methodology seems to be applicable to other substrates and reactions by choosing appropriate sensitizing crosslinkers and chiral building blocks.

\section{Photooxidation}

Díaz and co-workers established the potential of a various soft gels as nanoreactors for a model photocatalytic reaction (Scheme 11). ${ }^{41}$ Specifically, riboflavin tetraacetate (25)-catalyzed aerobic photooxidation of 1-(4-methoxyphenyl)ethanol (23) was investigated as a model reaction under blue visible light in gels made from either high or LMW compounds $(\mathbf{1 1}, \mathbf{1 4 - 2 2})$. Three strategies involving different degrees of organization of the photocatalyst within the gel network were explored, and the results were compared to those obtained in homogeneous and micellar solutions. In general, physical entrapment of both the catalyst and the substrate under optimized concentrations into the gels allowed the photooxidation with conversions between 55 and 100\% within $120 \mathrm{~min}$ (TOF $\sim 0.045-0.08 \mathrm{~min}^{-1} ; k \sim 0.011-0.028 \mathrm{~min}^{-1}$ ), albeit with first-order rates $c a$. 1-3-fold lower than in solution under comparable non-stirred conditions. Surprisingly, the organogel

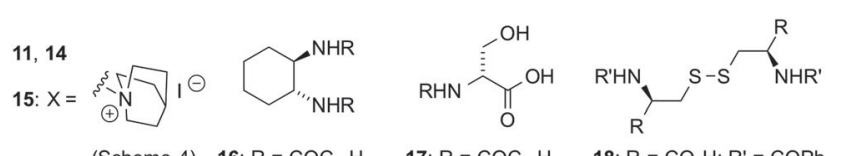
(Scheme 4) 16: $\mathrm{R}=\mathrm{COC}_{11} \mathrm{H}_{23} \quad$ 17: $\mathrm{R}=\mathrm{COC}_{11} \mathrm{H}_{23} \quad$ 18: $\mathrm{R}=\mathrm{CO}_{2} \mathrm{H} ; \mathrm{R}^{\prime}=\mathrm{COPh}$
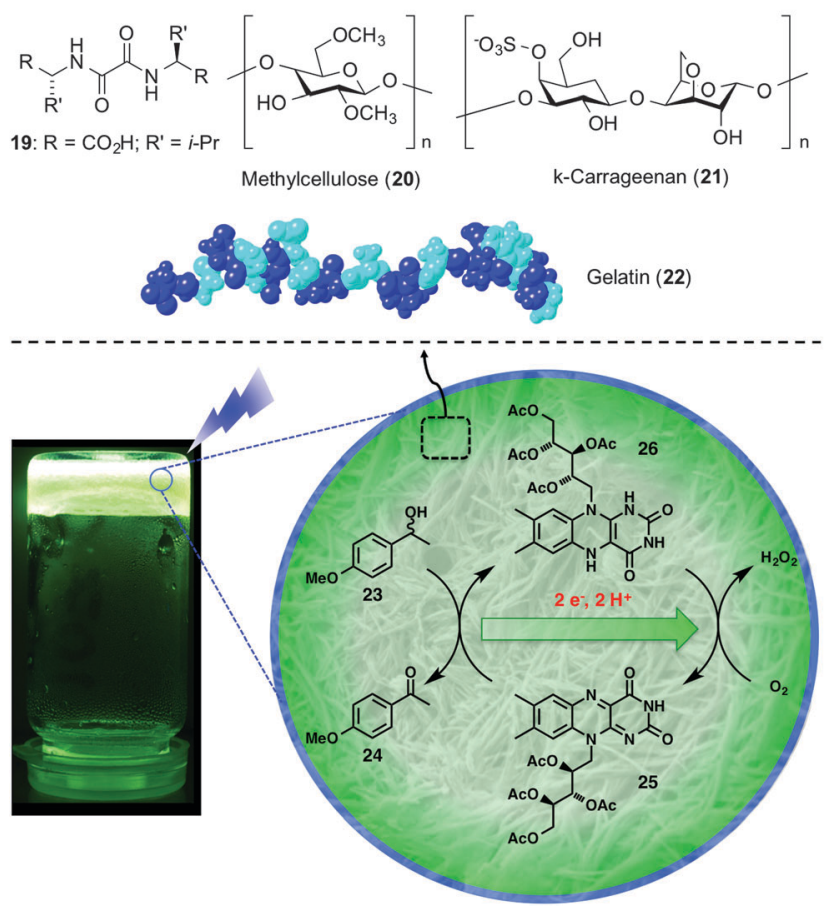

Scheme 11 Gelators (11, 14-22) (top) and riboflavin tetracetatecatalysed photooxidation of 1-(4-methoxymethyl)ethanol (23) inside gel media using blue visible light (bottom). Adapted with permission from ref. 41. Copyright (C) 2013 Royal Chemical Society.

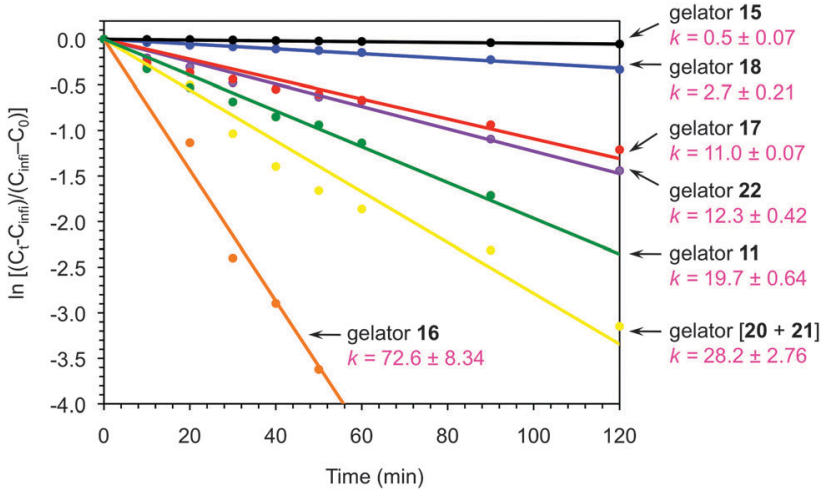

Scheme 12 Selection of first-order kinetics plots of RFT-catalyzed photooxidation of $\mathbf{2 3}$ in gel media prepared with different gelators. Solvent systems: $\mathrm{H}_{2} \mathrm{O}$ (gelators 17, 22, [20 + 21; $\left.1: 1 \mathrm{w} / \mathrm{w}\right]$ ); $\mathrm{H}_{2} \mathrm{O}+\mathrm{DMSO}(95: 5 \mathrm{v} / \mathrm{v})$ (gelator 18); $0.5 \mathrm{M} \mathrm{NaCl}$ (gelator 15); $\mathrm{H}_{2} \mathrm{O}+\mathrm{AcOH}(80: 20 \mathrm{v} / \mathrm{v}$ ) (gelator 11); $\mathrm{CH}_{3} \mathrm{CN}$ (gelator 16). Estimated rate constants $\left(\times 10^{3} \mathrm{~min}^{-1}\right), k$, are given for each case. Adapted with permission from ref. 41. Copyright (C) 2013 Royal Chemical Society.

made in $\mathrm{CH}_{3} \mathrm{CN}$ using the cyclohexane-based bisamide gelator 16 not only stabilized the catalyst against photodegradation but also afforded full conversion in less than $60 \mathrm{~min}$ (TOF $\sim 0.167 \mathrm{~min}^{-1}$; $k \sim 0.073 \mathrm{~min}^{-1}$ ) without the need of additional proton transfer mediators (e.g., thiourea) as it occurs in $\mathrm{CH}_{3} \mathrm{CN}$ solutions.

It is important to mention that the gelators could be recycled and reused in further runs without detriment to their gelation ability and reaction rates. Moreover, the kinetics of the photocatalytic process could be fine-tuned according to the properties of the gel media (Scheme 12). For example, entangled fibrillar networks with relatively high mechanical strength, as determined by scanning electron microscopy (SEM) and rheological measurements, were usually connected to lower reaction rates, whereas wrinkled laminated morphologies seemed to favor the reaction. In addition, the kinetic results showed in most cases a good correlation with the aeration efficiency of the gel media. ${ }^{41}$

\section{Conclusions and outlook}

Inspired by the efficiency of self-organizing compartmentation in natural systems to drive challenging transformations, research on photo-induced processes carried out inside gel matrices represents a growing and highly active new area (i.e., involving embedded reactants that do not participate directly in the assembly of the gel network). One of the advantages of photochemical processes for such studies is that it can be triggered without any thermal shock, which could disrupt the organization of the self-assembled fibrillar gel network. On the other hand, large active surface areas, good diffusion properties, variety of compositions, simple preparation from readily available molecular building blocks, very low concentration of gelator molecules, and the reversible stimuli-responsive nature of many self-assembled gels, make them ideal systems to mimic more complex natural systems.

A few initial studies on the rate of electron transfer photoreactions inside gels have provided the background for more 
demanding photophysical processes such as photon UC based on triplet-triplet annihilation (TTA-UC). Moreover, additional examples have demonstrated the feasibility of soft gels as reaction vessels and nanoreactors for photochemical processes such as photodimerizations, photosensitized isomerization, and photooxidations. Herein, the efforts are oriented towards the fabrication of spatially confined micro- and nanoreactors to improve kinetics, selectivity and/or processability in comparison to homogeneous solutions. Thus, important fields such as photovoltaics, photocatalysis and phototherapy will undoubtedly be great beneficiaries of future developments with respect to intragel photo-induced processes as well as the use of triple excited states as reporters.

Furthermore, a number of fundamental studies will still be necessary in future work in order to establish the real scope and boost the applicability of these materials as reaction vessels and nanoreactors for photo-induced processes. Those may include, among others, the following:

(a) The elucidation of different molecular interactions between the fibrillar network and the reactants that may play a key role in photo-induced processes. This will also shed light on the organization mode of reactants within the gel matrix,

(b) the correlation between gel properties (i.e., morphological, mechanical, diffusion, and thermodynamic properties) and the outcome of the photoreaction,

(c) the development of reliable strategies to improve the loading capacity of the gel (i.e., concentration of reactants) networks and their robustness for higher operational stability, while retaining their functional properties,

(d) the determination of optimal specificity and strength absorption of substrates in the gel matrix as well as the evaluation of possible concentration effects, and

(e) the fundamental understanding of mechanisms through which self-assembled gels may favor specific reaction pathways (e.g., steric interactions that could control spatial orientation of specific reactants and influence reaction selectivity, accumulation of reactants into nanostructured subdomains creating "hot-spots" of catalytic activity).

\section{Acknowledgements}

R.P.R thanks the financial support from the IEF Marie Curie program of the European Union (PIEF-GA-2013-625064). D.D.D. thanks the DFG for the Heisenberg professorship award (PRJ 9209720).

\section{Notes and references}

1 M. Vriezema, M. C. Aragonès, J. A. A. W. Elemans, J. J. L. M. Cornelissen, A. E. Rowan and R. J. M. Nolte, Chem. Rev., 2005, 105, 1445-1489.

2 Z. V. Todres, Organic chemistry in confined media, Springer International Publishing, Switzerland, 2013.

3 G. Palmisano, V. Augugliaro, M. Pagliaro and L. Palmisano, Chem. Commun., 2007, 3425-3437.
4 D. G. Shchukin and D. V. Sviridov, J. Photochem. Photobiol., C, 2006, 7, 23-39.

5 M. D. Ward, Chem. Soc. Rev., 1997, 26, 365-375.

6 A. Maldotti, A. Molinari and R. Amadelli, Chem. Rev., 2002, 102, 3811-3836.

7 M. Pagliaro, R. Ciriminna and G. Palmisano, Chem. Soc. Rev., 2007, 36, 932-940.

8 M. Antonietti and G. A. Ozin, Chem. - Eur. J., 2004, 10, 28-41.

9 J. H. Fendler, Chem. Rev., 1987, 87, 877-899.

10 J. G. Riess, Chem. Rev., 2001, 101, 2797-2920.

11 C. Harris and P. V. Kamat, ACS Nano, 2009, 3, 682-690.

12 O. V. Vasil'tsova and V. N. Parmon, Kinet. Catal., 1999, 40, 62-70.

13 K. Uekama, F. Hirayama and T. Irie, Chem. Rev., 1998, 98, 2045-2076.

14 D. G. Shchukin, E. A. Ustinovich, A. I. Kulak and D. V. Sviridov, Photochem. Photobiol. Sci., 2004, 3, 157-159.

15 D. K. Smith, in Supramolecular Chemistry From Molecules to Nanomaterials, ed. J. W. Steed and A. P. Gale, Wiley \& Sons Ltd., Chichester, UK, 1st edn, 2012, vol. 7, pp. 3355-3376.

16 D. D. Díaz, D. Kühbeck and R. J. Koppmans, Chem. Soc. Rev., 2011, 40, 427-448.

17 G. Perot and M. Guisnet, J. Mol. Catal., 1990, 61, 173-196.

18 M. N. Khan, Micellar catalysis, Surfactant science series, Boca Raton (FL), USA, CRC Press, 2007, vol. 133.

19 R. T. Cheriya, A. R. Mallia and M. Hariharan, Energy Environ. Sci., 2014, 7, 1661-1669.

20 A. Del Guerzo and J.-L. Pozzo, in Molecular Gels. Materials with Self-Assembled Fibrillar Networks, ed. P. Terech and G. Weiss, Kluwer Academic, New York, USA, 2005, pp. 813-851.

21 A. Shumburo and M. C. Biewer, Chem. Mater., 2002, 14, 3745-3750.

22 F. Galindo, M. I. Burguete, R. Gavara and S. V. Luis, J. Photochem. Photobiol., A, 2006, 178, 57-61.

23 M. I. Burguete, M. A. Izquierdo, F. Galindo and S. V. Luis, Chem. Phys. Lett., 2008, 460, 503-506.

24 P. D. Wadhavane, R. E. Galian, M. A. Izquierdo, J. AguileraSigalat, F. Galindo, L. Schmidt, M. Isabel Burguete, J. Pérez-Prieto and S. V. Luis, J. Am. Chem. Soc., 2012, 134, 20554-20563.

25 P. D. Wadhavane, M. A. Izquierdo, F. Galindo, M. I. Burguete and S. V. Luis, Soft Matter, 2012, 8, 4373-4381.

26 T. N. Singh-Rachford and F. N. Castellano, Coord. Chem. Rev., 2010, 254, 2560-2573.

27 J. Z. Zhao, S. M. Ji and H. M. Guo, $R S C A d v .$, 2011, 1, 937-950.

28 J. H. Kim and J. H. Kim, J. Am. Chem. Soc., 2012, 134, 17478-17481.

29 A. Monguzzi, R. Tubino, S. Hoseinkhani, M. Campione and F. Meinardi, Phys. Chem. Chem. Phys., 2012, 14, 4322-4332.

30 V. Gray, D. Dzebo, M. Abrahamsson, B. Albinsson and K. Moth-Poulsen, Phys. Chem. Chem. Phys., 2014, 16, 10345-10352.

31 R. Vadrucci, C. Weder and Y. C. Simon, Mater. Horiz., 2015, 2, 120-124. 
32 K. Sripathy, R. W. MacQueen, J. R. Peterson, Y. Y. Cheng, M. Dvořák, D. R. McCamey, N. D. Treat, N. Stingelind and T. W. Schmidt, J. Mater. Chem. C, 2015, 3, 616-622.

33 P. Duan, N. Yanai, H. Nagatomi and N. Kimizuka, J. Am. Chem. Soc., 2015, 137, 1887-1894.

34 A. Dawn, N. Fujita, S. Haraguchi, K. Sada and S. Shinkai, Chem. Commun., 2009, 2100-2102.

35 A. Dawn, N. Fujita, S. Haraguchi, K. Sada, S.-i. Tamaru and S. Shinkai, Org. Biomol. Chem., 2009, 7, 4378-4385.

36 A. Dawn, T. Shiraki, S. Haraguchi, H. Sato, K. Sada and S. Shinkai, Chem. - Eur. J., 2010, 16, 3676-3689.
37 S. Bhat and U. Maitra, Molecules, 2007, 12, 2181-2189.

38 Rajkamal, D. Chatterjee, A. Paul, S. Banerjee and S. Yadav, Chem. Commun., 2014, 50, 12131-12134.

39 S. Yadav, S. Banerjee, D. Maji and S. Lahiri, Tetrahedron, 2007, 63, 10979-10990.

40 X. Wei, W. Liang, W. Wu, C. Yang, F. Trotta, F. Caldera, A. Mele, T. Nishimoto and Y. Inoue, Org. Biomol. Chem., 2015, 13, 2905-2912.

41 J. Bachl, A. Hohenleutner, B. B. Dhar, C. Cativiela, U. Maitra, B. König and D. D. Díaz, J. Mater. Chem. A, 2013, 1, 4577-4588. 